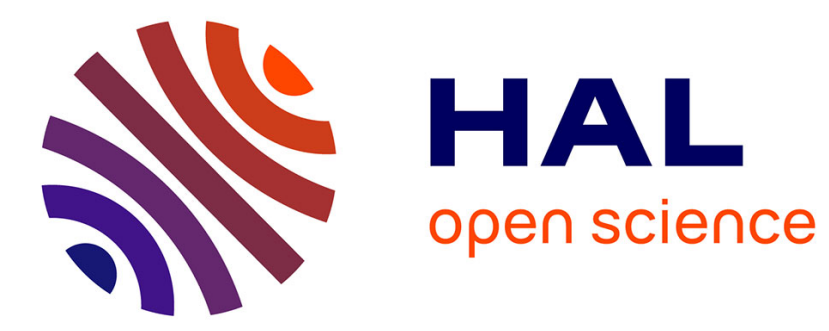

\title{
Electronically excited rubidium atom in a helium cluster or film.
}

\author{
Markku Leino, Alexandra Viel, Robert E. Zillich
}

\section{To cite this version:}

Markku Leino, Alexandra Viel, Robert E. Zillich. Electronically excited rubidium atom in a helium cluster or film.. Journal of Chemical Physics, 2008, 129 (18), pp.184308. 10.1063/1.3009279 . hal00675262

\section{HAL Id: hal-00675262 \\ https://hal.science/hal-00675262}

Submitted on $10 \mathrm{Jul} 2017$

HAL is a multi-disciplinary open access archive for the deposit and dissemination of scientific research documents, whether they are published or not. The documents may come from teaching and research institutions in France or abroad, or from public or private research centers.
L'archive ouverte pluridisciplinaire HAL, est destinée au dépôt et à la diffusion de documents scientifiques de niveau recherche, publiés ou non, émanant des établissements d'enseignement et de recherche français ou étrangers, des laboratoires publics ou privés. 


\title{
Electronically excited rubidium atom in a helium cluster or film
}

\author{
Markku Leino, ${ }^{1, a)}$ Alexandra Viel, ${ }^{1, b)}$ and Robert E. Zillich ${ }^{2, c)}$ \\ ${ }_{1}^{1}$ Institut de Physique de Rennes, UMR 6251, CNRS and Université de Rennes I, F-35042 Rennes, France \\ ${ }^{2}$ Institute for Theoretical Physics, Johannes Kepler Universität Linz, Altenbergerstraße 69, \\ A-4040 Linz, Austria
}

(Received 8 September 2008; accepted 8 October 2008; published online 12 November 2008)

\begin{abstract}
We present theoretical studies of helium droplets and films doped with one electronically excited rubidium atom $\mathrm{Rb}^{*}\left({ }^{2} P\right)$. Diffusion and path integral Monte Carlo approaches are used to investigate the energetics and the structure of clusters containing up to 14 helium atoms. The surface of large clusters is approximated by a helium film. The nonpair additive potential energy surface is modeled using a diatomic in molecule scheme. Calculations show that the stable structure of $\mathrm{Rb}^{*} \mathrm{He}_{n}$ consists of a seven helium atom ring centered at the rubidium, surrounded by a tirelike second solvation shell. A very different structure is obtained when performing a "vertical Monte Carlo transition." In this approach, a path integral Monte Carlo equilibration starts from the stable configuration of a rubidium atom in the electronic ground state adsorbed to the helium surface after switching to the electronically excited surface. In this case, $\mathrm{Rb}^{*} \mathrm{He}_{n}$ relaxes to a weakly bound metastable state in which $\mathrm{Rb}^{*}$ sits in a shallow dimple. The interpretation of the results is consistent with the recent experimental observations [G. Auböck et al., Phys. Rev. Lett. 101, 035301 (2008)]. () 2008 American Institute of Physics. [DOI: 10.1063/1.3009279]
\end{abstract}

\section{INTRODUCTION}

Helium nanodroplets offer a quite unique way to study a large variety of dopant molecules. ${ }^{1-4}$ These nanoscale clusters provide a very special matrix which combines cold temperature, quantum nature, and superfluidity, and they can be used to perform various kinds of spectroscopy. A large amount of theoretical and computational works about rotational and rovibrational spectra of molecules in helium have contributed substantially to our understanding of the solvation structure around the molecules, the molecule-helium coupling, and its relation to the ${ }^{4} \mathrm{He}$ collective excitations, see the reviews in Refs. 2-4. There is less theoretical work devoted to electronic excitations of molecules in helium but phenomenological treatments ${ }^{5}$ as well as quantum Monte Carlo simulations on different electronic surfaces ${ }^{6}$ have yielded encouraging results.

Within the variety of possible dopants, alkali atoms appear to be one of the best candidates to study electronic excitations due to their relatively simple electronic configuration with a single electron in the valence orbitals and the optically accessible electronic transitions. Experiments involving electronic excitations of one alkali atom adsorbed on a helium cluster are available. ${ }^{7-16}$ Some of them focused on the spectroscopy of helium- $\mathrm{Rb}$ exciplex formation. ${ }^{7-10}$ Theoretical modeling of these experiments is still a great challenge. However, a qualitative understanding of the exciplex formation has been partially achieved from the study of the potential energy surfaces of the different electronic states of the alkali atom but some aspects still need more theoretical effort. $^{9}$

\footnotetext{
${ }^{a)}$ Electronic mail: markku.leino@univ-rennes1.fr.

${ }^{b}$ Electronic mail: alexandra.viel@univ-rennes1.fr.

${ }^{c)}$ Electronic mail: robert.zillich@jku.at.
}

Due to the weak attractive interaction, alkali atoms in their ground electronic state are attached to the surface of helium clusters as established by density functional theory (DFT) (Ref. 17) and path integral Monte Carlo (PIMC) (Ref. 18) calculations. In this case, the interaction potential of the many-body problem can be modeled quite accurately within the pair potential approximation. For $p$-electronically excited alkali atoms, the interaction with helium atoms is no longer small and the pair approximation is no longer valid due to the anisotropic character of the interaction. The understanding of the solvation structure is thus nontrivial. One of the first question to answer is if the excited rubidium atom is inside or at the surface of the droplet. Since the simple pair potential approximation is not valid in this case, the answer is not obvious. Theoretical works dealing with excited alkali atoms in liquid helium, ${ }^{19,20}$ in solid helium, ${ }^{10,21-23}$ in ${ }^{4} \mathrm{He}$ cold gas ${ }^{24,25}$ as well as in clusters ${ }^{26}$ are available in the literature. They focus on determining the shape of the cavity in the liquid and the exciplex structures in solid and gas. Their conclusion is that exciplexes consist of a ring of helium atoms around the $p$-like electron density of the excited alkali atom. The number of helium atoms in the ring varies depending on the alkali species. Given the complexity of the systems, many of the calculations are based on some approximations. One of them is to assume a ring structure for the exciplex. Quantum mechanical studies of clusters doped with excited alkali atoms have been restricted to the work of Takayanagi and Shiga ${ }^{26}$ in which they studied $\mathrm{K}^{*} \mathrm{He}_{n}$ clusters for $n \leq 8$ using PIMC but neglecting the bosonic permutation. Bosonic permutations cannot be neglected when looking at the dynamic properties, which are what we want to study in future work. They have been included in this work.

Electronic excitation of an adsorbed rubidium atom has been the subject of experimental studies. ${ }^{7,9,12,13}$ It was shown 
that only the excitation to the ${ }^{2} \Pi_{3 / 2}$ state leads to the formation of a $\mathrm{Rb}^{*} \mathrm{He}$ exciplex. The absence of detection of exciplex formation after excitation to the lower ${ }^{2} \Pi_{1 / 2}$ state is interpreted by the presence of a potential energy barrier prohibiting the tunneling of a helium atom from the droplet toward the $\mathrm{Rb}$ atom. $\mathrm{Rb} \mathrm{H}^{*} \mathrm{He}$ exciplex formation has been theoretically studied by Reho et al. ${ }^{11}$ using a onedimensional semiclassical model parametrized using $\mathrm{Na}$ and $\mathrm{K}$ experimental data. ${ }^{12}$ The predictions of this model do not reproduce quantitatively the experiment of Droppelmann et $a l .{ }^{9}$ but are consistent with the nonobservation of exciplexes when exciting the lower ${ }^{2} \Pi_{1 / 2}$ state. Very recently, Auböck et $a l .{ }^{13}$ demonstrated using a combination of laser induced fluorescence and beam-depletion spectroscopy that a rubidium atom attached to a large helium droplet can be electronically excited to this lower electronic state without either being detached from the droplet or forming an exciplex. This quite unique result opened the route to electron spin pumping.

In Sec. II, the theoretical approach used to model helium clusters and films doped with one excited rubidium atom is presented. Section III focuses on clusters with up to $n=14$ helium atoms, while Sec. IV deals with a rubidium atom attached to a helium film. Finally, Sec. V summarizes the results.

\section{THEORETICAL APPROACH}

\section{A. Description of the system}

In this work, we consider clusters or films made of $n$ helium atoms and one rubidium atom which is in the electronic ground state $(\mathrm{Rb})$ or electronically excited state $\left(\mathrm{Rb}^{*}\right)$. The $3 n+3$ Cartesian coordinates of these $n+1$ atoms, $\mathcal{X}$, are used to model these systems. The corresponding Hamiltonian is given by

$$
\left(\begin{array}{cccc}
U_{11}+\Delta_{\text {SO }} & U_{11} & U_{12} & U_{12} \\
U_{11} & U_{11}-\Delta_{\text {SO }} & U_{12}+\sqrt{2} \Delta_{\text {SO }} & U_{12} \\
U_{12}^{*} & U_{12}^{*}+\sqrt{2} \Delta_{\text {SO }} & U_{22} & U_{22} \\
U_{12}^{*} & U_{12}^{*} & U_{22} & U_{22} \\
U_{13}^{*} & U_{13}^{*} & -U_{12}^{*} & -U_{12}^{*}+\sqrt{2} \Delta_{\text {SO }} \\
U_{13}^{*} & U_{13}^{*} & -U_{12}^{*} & -U_{12}^{*}
\end{array}\right.
$$

where $\Delta_{\mathrm{SO}}=237.6 \mathrm{~cm}^{-1}$ is the spin-orbit coupling constant for $\mathrm{Rb}$ and the matrix $U$ is defined by

$$
\begin{aligned}
& U_{11}=\sum_{i}\left[\frac{1}{2}\left(1+\cos ^{2} \theta_{i}\right) V_{\pi}\left(r_{i}\right)+\frac{1}{2} \sin ^{2} \theta_{i} V_{\Sigma}\left(r_{i}\right)\right], \\
& U_{22}=\sum_{i}\left[\sin ^{2} \theta_{i} V_{\pi}\left(r_{i}\right)+\cos ^{2} \theta_{i} V_{\Sigma}\left(r_{i}\right)\right],
\end{aligned}
$$

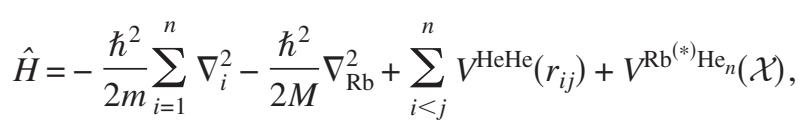

where $m=4.0026 \mathrm{u}$ is the mass of the helium atoms, $M$ $=84.91179 \mathrm{u}$ is the mass of the rubidium atom, and $r_{i j}$ is the distance between two helium atoms. Suitable periodic boundary conditions are added to Eq. (1) when studying one adsorbed $\mathrm{Rb}$ atom on He films. The term $\Sigma_{i<j} V^{\mathrm{HeHe}}\left(r_{i j}\right)$ is the commonly used pair potential approximation for the interaction between helium atoms for which we use the empirical potential by Aziz et al. ${ }^{27}$ For the interaction between Rb (i.e., in the electronic ground state) and helium, we also use a pair potential approximation, ${ }^{28}$ while a nonpairwise potential $V^{\mathrm{Rb}^{*} \mathrm{He}_{n}}$ accounts for the interaction between the excited rubidium atom $\mathrm{Rb}^{*}$ and the $n$ helium atoms. This interaction is modeled within a diatomic in molecule (DIM) approach. ${ }^{29}$ As described in detail in Ref. 18, the adiabatic potential energy surfaces are obtained by diagonalization of the $6 \times 6$ matrix representation of the operator $\hat{U}+\hat{V}_{\mathrm{SO}}$ in the $\left|l m_{l} s m_{s}\right\rangle$ basis with $l=1$ and $s=1 / 2$. The spin-orbit operator $\hat{V}_{\mathrm{SO}}$ is assumed to be independent of the intermolecular distance and is taken equal to the one of an isolated $\mathrm{Rb}$ atom, thus proportional to $l s$. The operator $\hat{U}$ is approximated by a sum of pair interactions between $\mathrm{Rb}^{*}$ in the ${ }^{2} P$ electronic state and each of the helium atoms.

The resulting complex matrix, expressed using the spherical coordinates, $r_{i}, \theta_{i}$, and $\phi_{i}$, of the $n$ helium atoms with respect to the rubidium atom in the space-fixed frame is given by

$$
\left.\begin{array}{cc}
U_{13} & U_{13} \\
U_{13} & U_{13} \\
-U_{12} & -U_{12} \\
-U_{12}+\sqrt{2} \Delta_{\mathrm{SO}} & -U_{12} \\
U_{11}-\Delta_{\mathrm{SO}} & U_{11} \\
U_{11} & U_{11}+\Delta_{\mathrm{SO}}
\end{array}\right)
$$

$$
\begin{aligned}
& U_{12}=\sum_{i} \frac{1}{\sqrt{2}} \sin \theta_{i} \cos \theta_{i} e^{-i \Phi_{i}}\left[V_{\pi}\left(r_{i}\right)-V_{\Sigma}\left(r_{i}\right)\right], \\
& U_{13}=\sum_{i} \frac{1}{2} \sin ^{2} \theta_{i} e^{-2 i \Phi_{i}}\left[V_{\pi}\left(r_{i}\right)-V_{\Sigma}\left(r_{i}\right)\right] .
\end{aligned}
$$

In Eqs. (3), $V_{\pi}$ and $V_{\Sigma}$ refer to the first and second excited states of the $\mathrm{Rb}^{*}-\mathrm{He}$ dimer correlating to $\mathrm{Rb}^{*}\left({ }^{2} P\right)$. In this work, we use the $a b$ initio potentials computed by 
Pascale ${ }^{28,30}$ using $l$-dependent pseudopotentials. This choice is motivated by the good agreement obtained by Brühl et al. ${ }^{7}$ using these $\mathrm{Rb}-\mathrm{He}$ potentials between the theoretical emission spectra and the observed fluorescence. This indicates that even if there is criticism of their accuracy, ${ }^{31,32}$ they are physically relevant. The diagonalization of the complex matrix Eq. (2) leads to three doubly degenerate potential energy surfaces due to Kramer's degeneracy. ${ }^{33}$ For the smallest cluster, $\mathrm{Rb}^{*} \mathrm{He}$, the effect of the spin-orbit coupling can been seen in Fig. 4 of Ref. 7. In addition to the lift of the degeneracy of $V_{\Pi}$, the lowest excited state $1^{2} \Pi_{1 / 2}$ presents both an attractive well and a barrier, the second excited state $1^{2} \Pi_{3 / 2}$ presents an attractive well only, and the upper state $2^{2} \Sigma_{1 / 2}$ is purely repulsive.

In this paper, we restrict our study to the first excited state of $\mathrm{Rb}^{*} \mathrm{He}_{n}$, thus the lowest eigenvalue of the matrix Eq. (2). This state is loosely referred to as the $1^{2} \Pi_{1 / 2}$ state using spectroscopic notation which is, in principle, only valid for linear molecules. The many-body system $\mathrm{Rb}^{*} \mathrm{He}_{n}$ described by Eq. (1) is simulated using zero temperature and finitetemperature Monte Carlo methods.

\section{B. Diffusion Monte Carlo}

In the present study, we use the importance sampling diffusion Monte Carlo (IS-DMC) method to evaluate the energy and structural properties of $\mathrm{Rb}^{*} \mathrm{He}_{n}$. Since DMC methodology is described in numerous publications, ${ }^{34-39}$ we focus here on the main features of the method and on technical details specific to its application to $\mathrm{Rb}^{*} \mathrm{He}_{n}$ systems.

In DMC with importance sampling, a guiding (or trial) function $\Psi_{T}(\mathcal{X})$ is introduced and the product $f(\mathcal{X}, \tau)$ $=\Phi(\mathcal{X}, \tau) \Psi_{T}(\mathcal{X})$ is propagated in imaginary time $\tau$ according to the diffusion equation for this $\mathcal{N}$-dimensional system ${ }^{34}$

$$
\begin{aligned}
\frac{\partial f(\mathcal{X})}{\partial \tau}= & \sum_{j=1}^{\mathcal{N}}\left\{D_{j} \frac{\partial^{2}}{\partial \mathcal{X}_{j}^{2}} f(\mathcal{X})-D_{j} \frac{\partial}{\partial \mathcal{X}_{j}}\left[f(\mathcal{X}) F_{j}(\mathcal{X})\right]\right\} \\
& -\left[E_{l}(\mathcal{X})-E_{\text {ref }}\right] f(\mathcal{X}),
\end{aligned}
$$

with $\mathcal{N}=3 n+3$. In the above equation, $\mathcal{X}$ is a vector in the $\mathcal{N}$-dimensional space, $D_{j}=\hbar^{2} / 2 M$ if $\mathcal{X}_{j}$ corresponds to a Cartesian coordinate of the rubidium atom, and $D_{j}=\hbar^{2} / 2 m$ if $\mathcal{X}_{j}$ corresponds to a Cartesian coordinate of a helium atom. The usual notation for the local energy $E_{l}(\mathcal{X})=\Psi_{T}(\mathcal{X})^{-1} \hat{H} \Psi_{T}(\mathcal{X})$ and for the quantum force $F_{j}(\mathcal{X})=\partial / \partial \mathcal{X}_{j} \ln \left|\Psi_{T}(\mathcal{X})\right|^{2}$ has been used. The energies are given with respect to the reference energy $E_{\text {ref }}$ such that the vibrational ground state of Eq. (1) is zero. The steady state of Eq. (4) is obtained via a random walk technique of an ensemble of walkers, i.e., an ensemble of vectors $\mathcal{X}$ specifying the position of the $n+1$ atoms. The short time approximation of the Green's function appropriate to Eq. (4) is numerically modeled by a random move sampled from a Gaussian distribution of width $\sqrt{2 D_{j} \Delta \tau}$, followed by a drift of $D_{j} \Delta \tau F_{j}(\mathcal{X}(\tau))$ for each of the $\mathcal{N}$ components of the walkers $\mathcal{X}$ in the ensemble. The imaginary time propagation is thus discretized with a discretization step, $\Delta \tau$. After this diffusion move, the source term $-\left[E_{l}(\mathcal{X})\right.$ $-E_{\text {refl }} f(\mathcal{X})$ needs to be evaluated. Different recipes to imple- ment this term exist. Either walkers carry continuous weights updated at each time step as

$$
w_{j}(\tau+\Delta \tau)=w_{j}(\tau) e^{-\left(E_{l}\left(\mathcal{X}_{j}\right)-E_{\mathrm{ref}}\right) \Delta \tau},
$$

or, in the pure branching scheme, walkers have a unit weight and are replicated or killed according to the value of $\exp \left[-\left(E_{l}\left(\mathcal{X}_{j}\right)-E_{\text {ref }}\right) \Delta \tau\right]$. The implementation used in this work relies on a combination of weights and branching, resulting in a fixed ensemble size ${ }^{40}$ similar to the implementation used in Ref. 41. It is described in detail in Ref. 42. Finally, a Metropolis step based on the trial function $\Psi_{T}$ is added at each time step. It forces an increased sampling in the regions where $\Psi_{T}$ is large.

There are two estimators for the ground state energy of Eq. (1). The first one is an average of the local energy over the walkers and the random walk, which will give the bosonic ground state energy in the limit of a large ensemble of walkers and of a small imaginary time step. The other energy estimate uses the variation in the total weight of the ensemble $W=\Sigma_{j} w_{j}$, after $p$ time steps,

$$
E_{\text {growth }}=E_{\text {ref }}+\frac{1}{p \Delta \tau} \ln \frac{W(\tau)}{W(\tau+p \Delta \tau)} .
$$

The resulting ground state energy is subject to time step and ensemble size bias, which must be carefully checked.

The computation of the expectation value of an operator $\hat{O}$, which does not commute with the Hamiltonian of the system, is not trivial. The calculation, via average over the ensemble of the expectation value of $\hat{O}$ from the asymptote $f(\mathcal{X}, \tau \rightarrow \infty)$, leads to the mixed estimator

$$
\frac{\sum_{i}^{N_{\mathrm{wlk}}} O\left(\mathcal{X}_{i}\right) w_{i}}{\sum_{i}^{N_{\mathrm{wlk}}} w_{i}} \approx \frac{\left\langle\psi_{T}(\mathcal{X})|\hat{O}| \phi(\mathcal{X})\right\rangle}{\left\langle\psi_{T}(\mathcal{X}) \mid \phi(\mathcal{X})\right\rangle}=\langle\hat{O}\rangle_{m},
$$

where $O\left(\mathcal{X}_{i}\right)$ corresponds to the evaluation of the operator $\hat{O}$ (most commonly a position operator) for the geometry of the walker $i$. The mixed estimate $\langle\hat{O}\rangle_{m}$ differs from the exact estimate $\langle\hat{O}\rangle_{P}=\langle\phi(\mathcal{X})|\hat{O}| \phi(\mathcal{X})\rangle$ as soon as the trial function $\psi_{T}$ differs from the exact ground state function $\phi$, which is always the case except for trivial problems. In order to compute the exact average with respect to $\phi$, the mixed estimate needs to be corrected by a multiplication by $\phi / \psi_{T}$ in the integrand of Eq. (7). This so-called "pure estimate" can be obtained by the descendent weighing scheme described in Refs. 43 and 44. We use the implementation in Ref. 43. The descendent weighing scheme is applied here to calculate probability densities from which the structural information can be inferred. The radial probability densities $\rho_{\text {rad }}^{\mathrm{RbHe}}$ between $\mathrm{Rb}$ and $\mathrm{He}$ and $\rho_{\text {rad }}^{\mathrm{HeHe}}$ between the helium atoms are the average of the operators

$$
\hat{\rho}_{\text {rad }}(R)=\frac{1}{n_{\text {pairs }}} \sum_{i=1}^{n_{\text {pairs }}} \frac{\delta\left(R_{i}-R\right)}{R^{2}} .
$$

Additional information on the structure of the cluster is obtained from the angular $\mathrm{He}-\mathrm{Rb}^{*}-\mathrm{He}$ distribution, which is the average of 


$$
\hat{\rho}_{\text {ang }}(\theta)=\frac{1}{n(n-1)} \sum_{i \neq j} \delta\left(\theta-\theta_{i j}\right),
$$

where $\theta_{i j} \in[0, \pi]$ is the angle between the directions from $\mathrm{Rb}$ to the $i$ th and to the $j$ th He atoms. In addition, the planarity of the cluster is also checked. For this, the leastsquares plane of all the $n+1$ atoms defined by its normal vector coefficients $a, b$, and $c$ is used. This plane is determined by solving the eigenvalue problem

$$
\left[\begin{array}{ccc}
a_{x x} & a_{x y} & a_{x z} \\
a_{y x} & a_{y y} & a_{x y} \\
a_{z x} & a_{z y} & a_{z z}
\end{array}\right]\left[\begin{array}{l}
a \\
b \\
c
\end{array}\right]=0,
$$

with the normalization condition $a^{2}+b^{2}+c^{2}=1$. The matrix elements $a_{i j}$, with $i, j \in\{x, y, z\}$ are the least-square distances, or covariances, given by

$$
a_{x y}=\sum_{i=1}^{n+1}\left(x_{i}-\langle x\rangle\right)\left(y_{i}-\langle y\rangle\right),
$$

for example. The three eigenvectors represent the "best," "intermediate," and "worst" planes. The corresponding eigenvalues are the sum of the squared distances to the "eigenplane." The planar character of the cluster is then measured by binning the lowest eigenvalue $d_{\min }$

$$
\hat{\rho}_{d}(d)=\delta\left(d_{\min }-d\right) .
$$

In IS-DMC, one relies on the quality of the trial function such that the source term $E_{l}(\mathcal{X})-E_{\text {ref }}$ in Eq. (4) is a smoother function of the geometry than the original potential term of Eq. (1), $\sum_{i<j}^{n} V^{\mathrm{HeHe}}\left(r_{i j}\right)+V^{\mathrm{Rb}^{*} \mathrm{He}}{ }_{n}(\mathcal{X})$. In this case, IS-DMC converges faster than an unbiased (i.e., a constant $\Psi_{T}$ ) DMC propagation by reducing the statistical fluctuations. However, the choice of the trial function must be done carefully in order not to bias the results, and physical insights are helpful in designing this function. A generic trial function, which respects the full permutation symmetry, can be written as ${ }^{45}$

$$
\Psi_{T}=\chi^{\mathrm{Rb}}\left(r_{\mathrm{Rb}}\right) \prod_{i \in \mathrm{He}} \chi^{\mathrm{He}}\left(r_{i}\right) \Phi^{\mathrm{HeRb}}\left(r_{i \mathrm{Rb}}\right) \prod_{i, j \in \mathrm{He}} \Phi^{\mathrm{HeHe}}\left(r_{i j}\right),
$$

in which $r_{\mathrm{Rb}}$ and $r_{i}$ are the distances of $\mathrm{Rb}$ and the $i$ th $\mathrm{He}$, respectively, to the center of the cluster, $r_{i \mathrm{Rb}}$ is the distance between the $\mathrm{Rb}$ atom and the $i$ th $\mathrm{He}$ atom, and $r_{i j}$ is the distance between two He atoms. In Eq. (13), Fermi-type [Eq. (14a)] and Jastrow-type [Eq. (14b)] functions are used

$$
\begin{aligned}
& \chi(r)=\left\{1+\exp \left[f_{1}\left(r-f_{2}\right)\right]\right\}^{-1}, \\
& \Phi(r)=\exp \left(-a r^{-5}\right) .
\end{aligned}
$$

$\chi(r)$ ensures asymptotic exponential decay without any structural bias at short range, while $\Phi(r)$ describes the short-range repulsion. In this work, we simplify the trial function to

$$
\Psi_{T}=\prod_{i \in \mathrm{He}} \chi^{\mathrm{He}}\left(r_{i}\right) \prod_{i, j \in \mathrm{He}} \Phi^{\mathrm{HeHe}}\left(r_{i j}\right),
$$

thus avoiding to bias the position of the rubidium atom with respect to the helium atoms. We stress that the use of a trial
TABLE I. Optimized $f_{1}$ and $f_{2}$ parameters of the trial function Eq. (13)

\begin{tabular}{llllll}
\hline \hline$n$ & $\leq 7$ & 8 & 9 & 12 & 14 \\
\hline$f_{1}$ & 1 & 0.5 & 0.4 & 0.3 & 0.3 \\
$f_{2}$ & 12 & 5 & 4 & 5 & 6 \\
\hline \hline
\end{tabular}

function for the study of $\mathrm{Rb}^{*} \mathrm{He}_{n}$ is mandatory for $n \geq 8$. Indeed for $n \geq 8$, an unbiased DMC leads to unphysical $\mathrm{He}$ evaporation frequently encountered in DMC studies of weakly bound systems. The parameter $a$ has been taken equal to $500 a_{0}^{5}$ as advised in Ref. 45 in which the value $a$ $\approx 3000 a_{0}^{5}$ adapted to pure $\mathrm{He}$ clusters has been reduced to $500 a_{0}^{5}$ in order to model a strong $\mathrm{Ne}^{+} \mathrm{He}_{2}$ core.

IS-DMC yields the exact ground state energy in the limit of infinite propagation time, infinite ensemble size, and vanishing time step $\Delta \tau$. The statistical fluctuations can be reduced by optimizing the trial wave function. The optimization of parametrized trial functions is usually done using a variational Monte Carlo (VMC) study. Indeed VMC obeys the variational principle, thus the average of the local energy is an upper bound of the exact energy, and the associated variance vanishes if the trial function approaches the exact ground state. In our case, the trial function ansatz does not act on the rubidium atom position. Thus a VMC computation is not possible (the position of the $\mathrm{Rb}$ atom is not correlated with the position of the helium atoms) and it is thus of no use to optimize the parameters of the trial function. Instead ISDMC runs are used to optimize the trial function parameters by examining the energy variance. Only the two parameters, $f_{1}$ and $f_{2}$, of the Fermi-type function Eq. (14a) have been optimized for each cluster size studied. Both the variance and the energy are inspected during the optimization process. The resulting parameters used are given in Table I. Attention has been paid to the time step bias due to the Trotter expansion of the propagator.

\section{PIMC}

The PIMC method is based on Feynman's original idea to represent quantum dynamics by paths in configuration space. ${ }^{46}$ Applied to quantum statistical mechanics, it leads to an isomorphism between quantum theory and a classical system of closed polymer chains. ${ }^{47}$ For bosons, such as ${ }^{4} \mathrm{He}$ atoms, PIMC is an exact finite-temperature method if Bose exchange symmetry, which is responsible for all the interesting quantum effects such as superfluidity or Bose Einstein condensation, is taken into account by allowing these polymers to reconnect to form larger polymers. Superfluidity can then be quantified in terms of macroscopic exchange-coupled paths that are comparable to the system size.

In short, the PIMC method is an application of the Metropolis algorithm ${ }^{48}$ to the configuration space representation of the many-body density matrix

$$
\rho\left(\mathcal{X}, \mathcal{X}^{\prime} ; \beta\right)=\left\langle\mathcal{X}\left|e^{-\beta \hat{H}}\right| \mathcal{X}^{\prime}\right\rangle,
$$

where $\beta=1 / k_{B} T$. For the evaluation of $\rho\left(\mathcal{X}, \mathcal{X}^{\prime} ; \beta\right)$, the "imaginary time" interval $\beta$ is split into smaller time steps 
$\tau=\beta / M$ which necessitates the introduction of new coordinates at intermediate time slices,

$$
\begin{aligned}
\rho\left(\mathcal{X}_{0}, \mathcal{X}_{M} ; \beta\right)= & \int d \mathcal{X}_{1} \cdots \mathcal{X}_{M-1} \\
& \times \rho\left(\mathcal{X}_{0}, \mathcal{X}_{1} ; \tau\right) \cdots \rho\left(\mathcal{X}_{M-1}, \mathcal{X}_{M} ; \tau\right)
\end{aligned}
$$

In the above equation, $\left(\mathcal{X}_{0}, \ldots, \mathcal{X}_{M}\right)$ is a discretized path in imaginary time. For sufficiently small $\tau, \rho\left(\mathcal{X}_{0}, \mathcal{X}_{1} ; \tau\right)$ can be approximated in various ways such as the Trotter ("primitive") approximation

$$
e^{-\tau \hat{H}}=e^{-\tau \hat{V} / 2} e^{-\tau \hat{T}} e^{-\tau \hat{V} / 2}+\mathrm{O}\left(\tau^{3}\right),
$$

higher order approximations based on the Campbell-BakerHausdorff expansion ${ }^{49,50}$ or the pair density approximation. ${ }^{51}$ If only averages of diagonal operators are required (such as energy or density distributions), we set $\mathcal{X}_{M}=\mathcal{X}_{0}$. Finally, Bose symmetry is implemented by symmetrization of the density matrix

$$
\rho_{B}(\mathcal{X}, \mathcal{X} ; \beta)=\frac{1}{N !} \sum_{P} \rho(\mathcal{X}, P \mathcal{X} ; \beta)
$$

which, in the Metropolis sampling scheme, corresponds to reconnecting the imaginary time paths to form larger chains. An excellent review of the PIMC method for bosons can be found in Ref. 51. Applications of PIMC to doped helium-4 can be found in Refs. 52 and 53.

All PIMC simulations are done at $T=0.31 \mathrm{~K}$, which corresponds approximately to the experimentally determined temperature of ${ }^{4} \mathrm{He}$ droplets generated by nozzle expansion. When possible, the pair density approximation is used rather than the primitive approximation for the imaginary time Green's function $\exp (-\tau \hat{H})$, which is only second order in the imaginary time step. For the $\mathrm{He}-\mathrm{He}$ interaction and the $\mathrm{Rb}-\mathrm{He}$ interaction, if $\mathrm{Rb}$ is in the electronic ground state, we use the pair density approximation. However, since the interaction $\mathrm{Rb}^{*}-\mathrm{He}_{n}$ is not pairwise additive, we have to use the primitive approximation when studying the electronically excited cluster. Since this typically induces a larger time step bias than higher order approximations, we carefully check the convergence of our results with respect to $\tau$.

\section{RESULTS FOR Rb*He ${ }_{n}$ CLUSTERS}

In this section, we focus on small helium clusters doped by one electronically excited $\mathrm{Rb}$ atom. Both DMC and PIMC studies are presented after an analysis of the nontrivial $\mathrm{Rb}^{*}-\mathrm{He}_{n}$ potential energy surface for the $1^{2} \Pi_{1 / 2}$ state of rubidium.

\section{A. Potential energy surface}

The potential energy surface of the $\mathrm{Rb}^{*} \mathrm{He}_{n}$ clusters is the result of a diagonalization of the $6 \times 6$ matrix given in Eq. (2). Therefore, its shape is not trivial and it is interesting to study, for example, the geometry of the minimum as a function of $n$. For the smallest nontrivial case, $n=2$, the surface is visualized by the cut shown in Fig. 1 to give some first insights into the possible structure of the clusters. In this

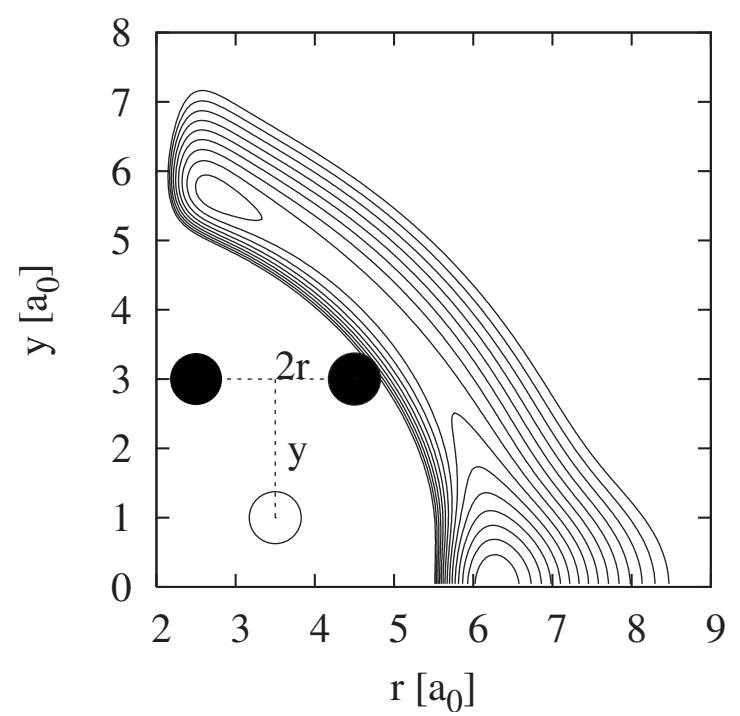

FIG. 1. Two-dimensional cut of the potential energy surface of $\mathrm{Rb}^{*} \mathrm{He}_{2}$ cluster along the simultaneous displacement of the two He atoms spotted by the distance between them $(2 r)$ and the distance $(y)$ of their center of mass to the $\mathrm{Rb}$ atom fixed at the origin (as pictured in the inset). The minimum of potential of $-349.9 \mathrm{~cm}^{-1}$ occurs for a linear geometry. The secondary minimum, which corresponds to a bent geometry $\left(r=2.7 a_{0}\right.$ and $\left.y=5.65 a_{0}\right)$, is $60 \mathrm{~cm}^{-1}$ higher in energy. The equipotential lines are $10 \mathrm{~cm}^{-1}$ apart.

figure, equipotential curves in the two-dimensional $(r, y)$ plane for isosceles $\mathrm{Rb}^{*} \mathrm{He}_{2}$ geometries are shown. The global minimum of the surface occurs at a linear geometry $(y=0)$ for a distance between $\mathrm{He}$ and $\mathrm{Rb}$ of $r=6.2 a_{0}$ in good agreement with the values given by Hirano et al..$^{31}$ and Hofer et $a l{ }^{10}$ This linear minimum configuration differs from the case of $\mathrm{K}^{*} \mathrm{He}_{2}$ for which the minimum of energy corresponds to a bent geometry. This nonlinear minimum for $\mathrm{K}^{*} \mathrm{He}_{2}$, which is obtained using the $\mathrm{K}-\mathrm{He}$ interaction curves from Pascale ${ }^{28,30}$ together with a spin-orbit coupling constant $\Delta_{\text {SO }}$ $=55.72 \mathrm{~cm}^{-1}$, can been seen in the angular density computed by Takayanagi and Shiga ${ }^{26}$ using a path integral approach. This difference between $\mathrm{Rb}$ and $\mathrm{K}$ can be removed by artificially lowering the spin-orbit coupling of $\mathrm{Rb}$ down to $\Delta_{\mathrm{SO}}=3 \mathrm{~cm}^{-1}$. A secondary minimum of $\mathrm{Rb}^{*} \mathrm{He}_{2}$ occurs for a bent geometry and is approximately $60 \mathrm{~cm}^{-1}$ above the global minimum (see Fig. 1 at $r=2.7 a_{0}$ and $y=5.65 a_{0}$ ). The geometry of this local minimum is similar to the geometry of the global minimum when $\Delta_{\mathrm{SO}}$ is artificially reduced. Inspection of the $(r, y)$ cut of the surface close to the potential minimum reveals a rather harmonic shape for which one could expect that a normal mode analysis will give a reasonable estimate of the zero point energy (ZPE).

A study of the global minimum of the potential energy surface for increasing number of $\mathrm{He}$ atoms in the cluster shows that the geometry of the minimum for eight $\mathrm{He}$ atoms is an eightfold ring centered at the $\mathrm{Rb}$ atom. Planar ring geometries for $\mathrm{Rb}^{*} \mathrm{He}_{n}$, defined by $r_{i}=r, \theta_{i}=2 \pi / n$ with $r_{i}$ and $\theta_{i}$ being the polar coordinates of the $i$ th $\mathrm{He}$ atom with respect to $\mathrm{Rb}$, have been studied in solid helium. ${ }^{10}$ The potential energy curves as a function of $r$ for such planar rings with $n=2-9$ are Morse-type functions with a single minimum. Figure 2 presents the evolution of the minimum geometry and energy as a function of $n$. For $n \leq 8$, the minimum 


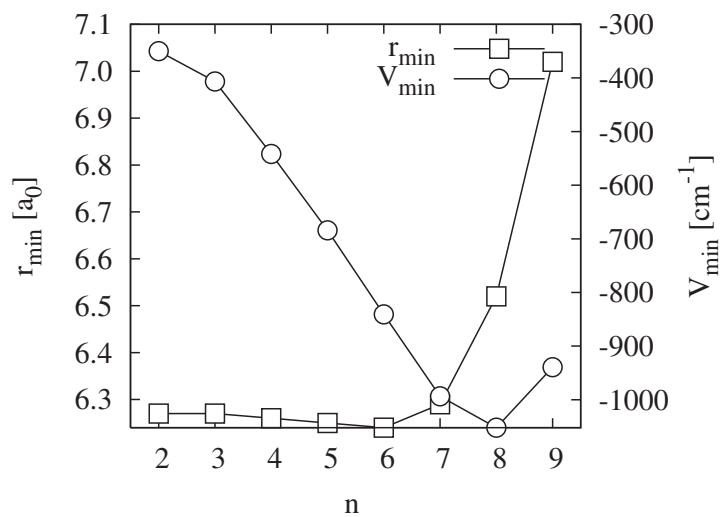

FIG. 2. Position $r_{\min }$ (in $a_{0}$ ) and value $V_{\min }\left(\right.$ in $\mathrm{cm}^{-1}$ ) of the minimum of potential along the radius $r$ of the regular ring (see text) as a function of the number of $\mathrm{He}$ atoms $n$.

energy value along the cut is a decreasing function of $n$, thus $\mathrm{Rb}^{*} \mathrm{He}_{n}$ is more stable than $\mathrm{Rb}^{*} \mathrm{He}_{n-1}$. Simultaneously, the optimized radius $r_{\min }$ of the ring stays nearly constant, with an increase for $n=8$. For $n=9$, the situation changes with a higher energy for the minimum and a much larger $r_{\min }$. This is a consequence that for $n=9$ the conformation of the global minimum is no longer a regular ring.

The energy of the global minimum, without any constraint, is presented in Fig. 3 as a function of $n$. We find that although there are multiple minimum energy configurations for large $n$, the optimization works very reliably and yields consistent minimum potential energies. A normal mode analysis is performed for these geometries, and the corresponding ground state energies within the harmonic approximation are also presented in Fig. 3. The computed harmonic ground state energies are much higher than the classical minima due to the large zero point motion of the light ${ }^{4} \mathrm{He}$ atoms. In addition, the $n$-dependence is nonmonotonic and a minimum is observed for $\mathrm{Rb}^{*} \mathrm{He}_{7}$, leading to the unphysical result that the chemical potential would be positive for the 8th He atom. Hence, within the harmonic approximation, $\mathrm{Rb}^{*} \mathrm{He}_{n}$ would exist only for $n \leq 7$. A similar nonmonotonic behavior has been reported in Refs. 10 and 31. The normal

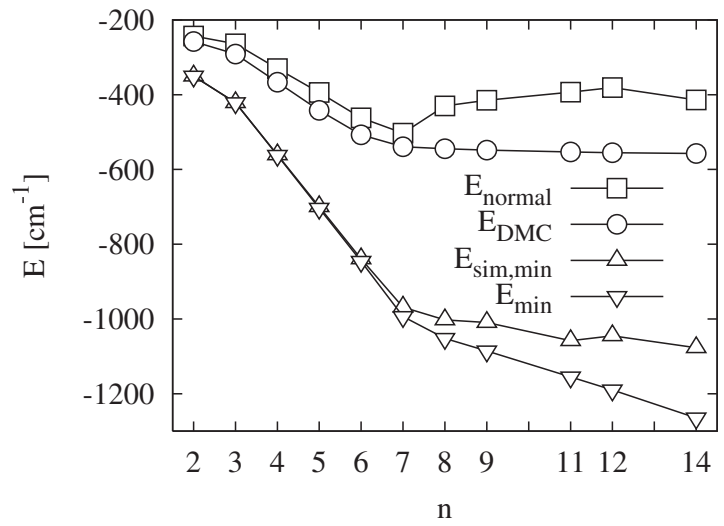

FIG. 3. Evolution of the global minimum energy (down triangle), of the harmonic estimate of the ground state energy level (square), of the minimum of potential sampled during the MC walk (up triangle), and of the DMC ground state energy (circle) as a function of $n$. Energies are given in $\mathrm{cm}^{-1}$. The error bars associated to the DMC energies are smaller than the symbol size.
TABLE II. Vibrational ground state energies computed using the IS-DMC method as a function of $n$. The average chemical potential $\bar{\mu}(n)$ as defined in the text and the ZPE are also given. Energies are given in $\mathrm{cm}^{-1}$.

\begin{tabular}{ccrr}
\hline \hline$n$ & \multicolumn{1}{c}{$E_{\text {growth }}$} & $-\bar{\mu}(n)$ & ZPE \\
\hline 2 & $-257.75 \pm 0.005$ & $\ldots$ & 92.3 \\
3 & $-291.32 \pm 0.008$ & 33.6 & 130.2 \\
4 & $-366.66 \pm 0.008$ & 75.3 & 195.5 \\
5 & $-441.66 \pm 0.01$ & 75.0 & 262.0 \\
6 & $-507.41 \pm 0.02$ & 65.7 & 338.3 \\
7 & $-539.35 \pm 0.05$ & 31.9 & 454.2 \\
8 & $-544.53 \pm 0.04$ & 5.2 & 507.5 \\
9 & $-548.1 \pm 0.1$ & 3.6 & 537.5 \\
11 & $-553.0 \pm 0.1$ & 2.5 & 602.2 \\
12 & $-555.3 \pm 0.1$ & 2.3 & 634.6 \\
14 & $-557.4 \pm 0.1$ & 1.1 & 707.6 \\
\hline \hline
\end{tabular}

mode analysis performed in Ref. 10 yielded a minimum for $n=6$. However, in this study, the interaction potential is chosen to reproduce a regular ring structure. In Ref. $31, n=6$ is also found to correspond to the minimum of the vibrational ground state energy as a function of $n$. In that work, ${ }^{31}$ the ring structure was also enforced, $V_{\pi}$ and $V_{\Sigma}$ interaction potentials for $\mathrm{Rb}-\mathrm{He}$ differed from the ones from Pascale ${ }^{28,30}$ and a more elaborate way to compute the vibrational ground state energy were used. The difference by one $\mathrm{He}$ atom for the minimum is certainly due to the different potential energy functions used. In addition, we found that the geometry of the minimum for $n=6$ is not a ring of sixfold symmetry. Thus the normal mode analysis we are presenting here is not performed at the same geometry as in Ref. 31. We conclude that even if the harmonic approximation is reasonable for up to seven $\mathrm{He}$ atoms, it is definitely not an appropriate approach for larger $n$, as we will also see in Sec. III B.

\section{B. DMC and PIMC results for small clusters}

The exact vibrational ground state energies computed using IS-DMC (see Sec. II) are reported in Fig. 3. An ensemble of 1000 walkers and a time step of $\Delta \tau=10$ a.u. have been used. Depending on the cluster size, 3000-50 000 blocks of 2000-8000 time steps have been performed after relaxation of the ensemble. The exact energies are quite similar to the harmonic approximation values for cluster sizes $n$ $\leq 7$ but strongly deviate for larger $n$. The exact energies are always found to be lower than the energies obtained within the normal mode approximation, highlighting the anharmonicity of the interaction potential. The variation in the exact energies as a function of $n$ is found to be monotonic, leading to a negative average chemical potential defined by $\bar{\mu}$ $=[E(n)-E(m)] /(n-m)$ given in Table II. The first seven He atoms appear to be strongly bound as inferred from the slope of the curve in Fig. 3 and from the chemical potential (Table II). As shown below, they form a rigid "core ring" around the $\mathrm{Rb}$ atom. For $n \geq 8$, the He atoms are more weakly bound and are located further away from $\mathrm{Rb}$.

In Fig. 3, we also present the minimum of the potential energy sampled during the IS-DMC simulation, i.e., the lowest value of the potential energy surface encountered during the Metropolis random walk. ${ }^{45}$ Notice that except for the 


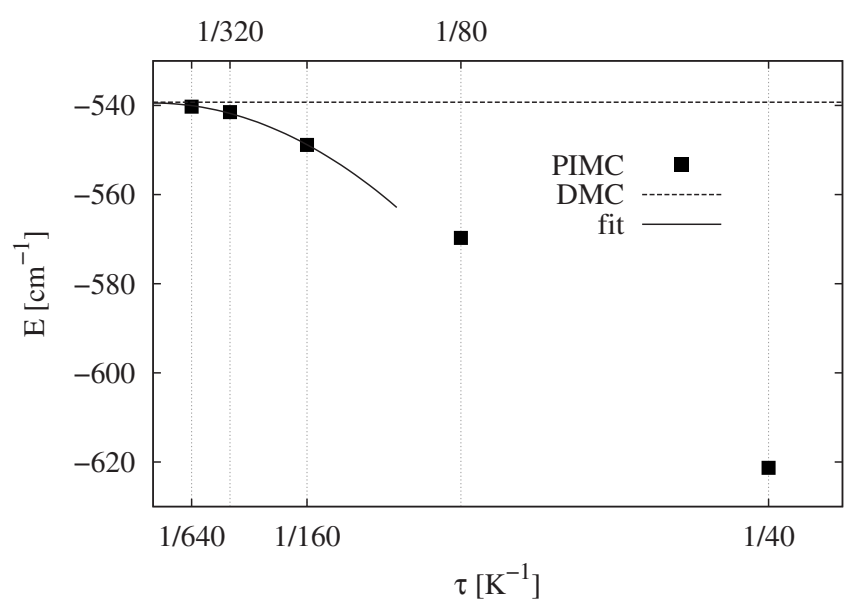

FIG. 4. PIMC time step dependence of the energy of $\mathrm{Rb}^{*} \mathrm{He}_{7}$ cluster obtained via PIMC at $T=1.25 \mathrm{~K}$. Error bars are within the symbol size. The DMC energy of $-539.3 \mathrm{~cm}^{-1}$ (dashed horizontal line) is obtained using $E_{\text {growth }}$ estimate with 1000 walkers and $\Delta \tau=10$ a.u.. The full line is a quadratic fit to the energies for small $\tau$, with an extrapolated value of $-539.5 \pm 0.3 \mathrm{~cm}^{-1}$.

small clusters, it is higher in energy than the absolute minimum of the potential. This could be an effect of the geometric increase in dimensionality of the problem, while sampling time and walker ensemble size are finite. However, it could also be because the amplitude of the wave function is small at the potential minimum. For $n \geq 8$, this assumption of small amplitude of the wave function at the geometry of the potential minimum is reasonable since the true average geometry contains a ring of seven helium atoms, while the classical potential minimum has eight atoms.

The DMC energy obtained for the $\mathrm{Rb}^{*} \mathrm{He}_{7}$ cluster is compared to the value given by PIMC in Fig. 4. In PIMC, the time step bias is mostly due to the strong nonpairwise interaction between $\mathrm{Rb}^{*}$ and the He atoms, which imposes the use of the primitive approximation. The zero time step limit of the quadratic fit based on the three smallest time steps is in good agreement with the DMC energy. Figure 4 stresses that careful check of the time step bias must be performed when extracting physical quantities from a PIMC simulation when the primitive approximation is used.

Insights into the structure of the clusters can be obtained from distribution functions using the descendent weighing scheme $^{43}$ (see Sec. II) to obtain pure estimators. Figure 5 shows the $\mathrm{Rb}-\mathrm{He}$ radial density distribution for $n=2,3,6,7$, 8 , and 14 . For $n \leq 7$, the distributions look very similar, consisting of a single peak with similar maximum and width. The distribution is asymmetric, reflecting the anharmonicity of the potential. For $n=8$, a second peak appears at larger distances, thus leading to the conclusion that the first seven He atoms form an inner "shell," whereas the 8th is farther away. As shown below, the seven He atoms in fact form a ring similar to the ring observed for the minimum of the potential energy surface. Note, however, that the He-He repulsion together with the ZPE lead to a smaller maximum number of $\mathrm{He}$ atoms in the ring. Indeed the classical minimum can accommodate eight He atoms, whereas the vibrational ground state consists of a ring of seven $\mathrm{He}$ atoms (core ring) with the extra He atoms farther away from the Rb. This

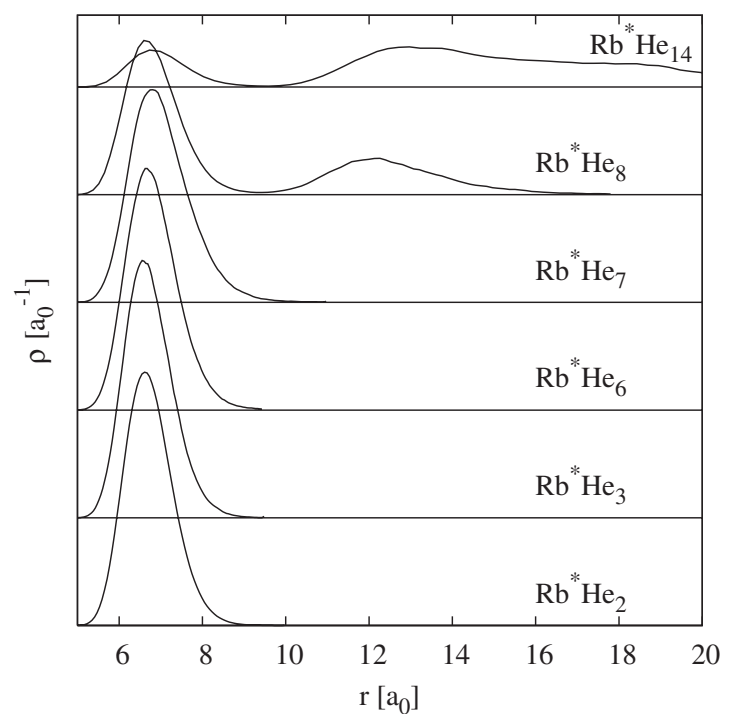

FIG. 5. Rb-He pair density distribution for selected cluster sizes as a function of the $\mathrm{Rb}-\mathrm{He}$ distance in $a_{0}$. The curves are vertically shifted to allow better reading.

strong difference in geometry for $\mathrm{Rb}^{*} \mathrm{He}_{8}$ between the classical and the quantum result is the reason of the severe failure of the normal mode approximation for clusters with eight or more $\mathrm{He}$ atoms.

Figure 6 shows the He-He pair distributions. For $n=2$, the distribution is a single peak whose maximum is roughly twice the one observed in Fig. 5, in agreement with a linear geometry of the $\mathrm{Rb}^{*} \mathrm{He}_{2}$ potential minimum. For $3 \leq n \leq 7$, the distributions have two broad overlapping peaks and include shorter $\mathrm{He}-\mathrm{He}$ distances. The minimum between the two peaks becomes more pronounced as $n$ increases to seven, thus indicating a more rigid structure for $n=7$. For the larger clusters, the short $\mathrm{He}-\mathrm{He}$ distribution is similar to the $n=7$ case and, in addition, larger $\mathrm{He}-\mathrm{He}$ distances are seen.

The He-He pair distributions in Fig. 6 do not provide an unambiguous picture of the structure of the $\mathrm{Rb}^{*} \mathrm{He}_{n}$ clusters.

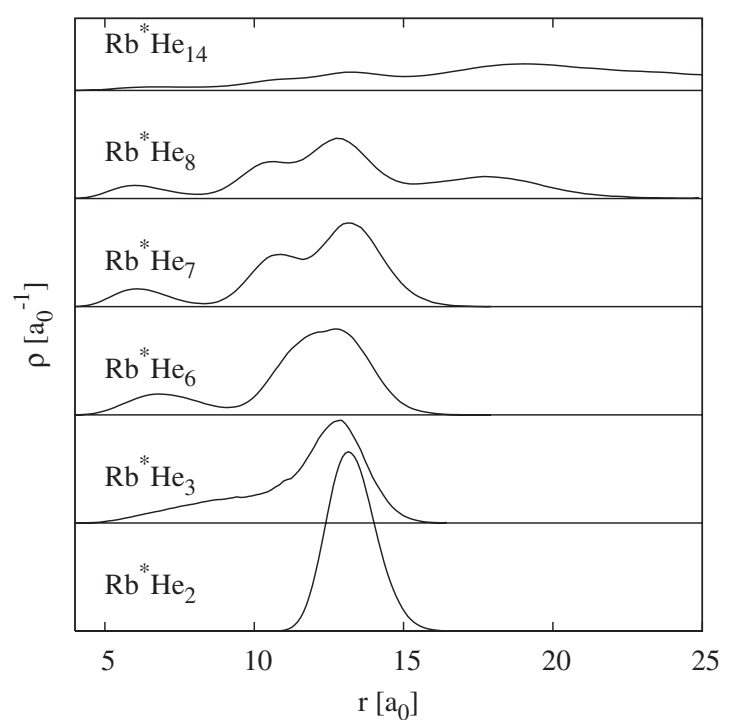

FIG. 6. He-He pair density distribution for selected cluster sizes as a function of the He-He distance in $a_{0}$. A vertical shift in the distribution is used to allow better reading. 


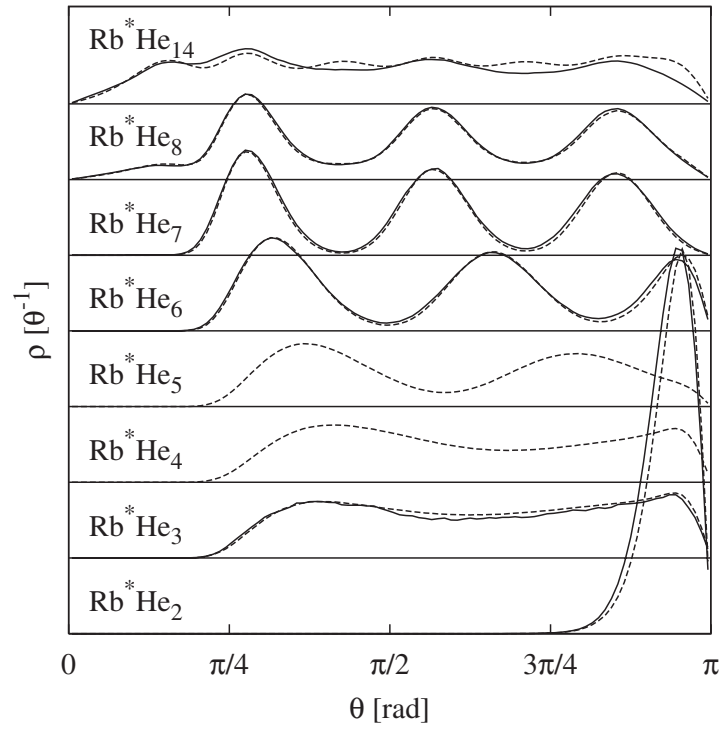

FIG. 7. He-Rb-He angular density distribution $\rho(\theta)$ for selected cluster sizes as a function of the angle in radians. IS-DMC pure estimates (full lines) and PIMC distributions (dashed lines) are shown with a vertical shift to ease readability.

A clearer picture is provided by the angular distribution $\rho(\theta)$, as defined in Eq. (9), which is shown in Fig. 7. Also shown are the results obtained at finite temperature of $T=0.31 \mathrm{~K}$ via PIMC, which are in excellent agreement with the pure DMC estimates at $T=0 \mathrm{~K}$. In Fig. 7 we use a PIMC time step of $\tau=1 / 40 \mathrm{~K}^{-1}$ which, in light of Fig. 4, is certainly too large to obtain meaningful energies. However, reducing the time step to $\tau=1 / 80 \mathrm{~K}^{-1}$ does not change $\rho(\theta)$ within the error bars; hence such a large PIMC time step is indeed sufficient to get accurate results for $\rho(\theta)$. The reason for such a small time step bias is that $\rho(\theta)$ is mostly determined by the $\mathrm{He}-\mathrm{He}$ interaction, for which we use the superior pair density approximation. ${ }^{51}$ A time step of $\tau=1 / 40 \mathrm{~K}^{-1}$ is found to be adequate when studying pure helium systems with PIMC for which the pair density approximation is used. ${ }^{51}$ We note that for other quantities such as the $\mathrm{Rb}-\mathrm{He}$ pair distribution, the time step bias would be larger.

For $n=2, \rho(\theta)$ is peaked at the linear geometry. Note that $\rho(\theta)$ has to vanish for $\theta=0$ and $\theta=\pi$ due the geometry factor $\sin \theta$ implicit in the definition given in Eq. (9). For $n=3$ and 4 , the distribution is quite broad with nearly no structure indicating floppy clusters. As $n$ further increases, $\rho(\theta)$ attains a more pronounced structure indicating an increase in rigidity. The $\mathrm{Rb}^{*} \mathrm{He}_{7}$ cluster appears to be the most rigid, as $\rho(\theta)$ nearly goes to zero between the maxima. This structure of $\rho(\theta)$ is consistent with the formation of a rigid ring of seven He atoms for which three well-isolated peaks are expected. For $n \geq 8, \rho(\theta)$ does not vanish anymore, consistent with the formation of a second "solvation shell." The minimum of the potential for the most rigid $\mathrm{Rb}^{*} \mathrm{He}_{7}$ cluster is a sevenfold ring with $\mathrm{He}-\mathrm{He}$ distances of 5.5, 9.8, and $12.3 a_{0}$. Although the zero point motion broadens the peaks considerably, the same distance pattern is observed in $\rho(\theta)$. Using the scheme proposed in Ref. 54 to correct for the overall rotation of the cluster as well as the motion of particles in imaginary time, we calculate the three-dimensional $\mathrm{He}$ density of $\mathrm{Rb}^{*} \mathrm{He}_{7}$

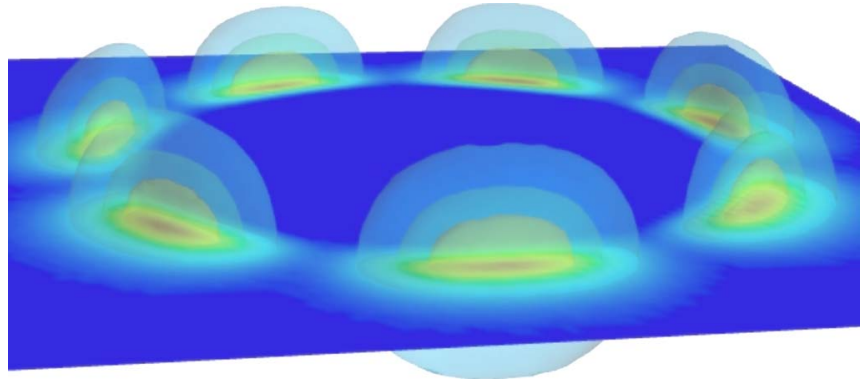

FIG. 8. (Color online) Surfaces of constant ${ }^{4} \mathrm{He}$ density for the $\mathrm{Rb}^{*} \mathrm{He}_{7}$ cluster and a false color map of the density in the $z=0$ plane are shown.

with respect to $\mathrm{Rb}^{*}$. In Fig. 8 we show surfaces of constant density as well as a false color plot of the density in the plane $z=0$. The locations of seven well-separated $\mathrm{He}$ atoms can clearly be discerned. For the largest cluster shown in Fig. 7, $\mathrm{Rb}^{*} \mathrm{He}_{14}$, we observe additional intermediate peaks in $\rho(\theta)$. Closer inspection shows that around the core ring of seven atoms there is a secondary ring of seven atoms which is commensurate with the core ring. Although it is more floppy than the core ring and has a considerably larger circumference, the atoms in the secondary ring sit preferably in the "grooves" of the core ring.

The most interesting and unusual feature of the $\mathrm{Rb}^{*} \mathrm{He}_{n}$ clusters is the fact that they are not spherical. Indeed, when looking at the distance between the $n \mathrm{He}$ atoms to the mean plane, introduced in Sec. II, we see that the clusters are indeed very flat. In Fig. 9 we show the probability distribution of the average distance of $\mathrm{He}$ atoms from the mean plane. For example, in the case of the $n=7$ cluster, this distance to the mean plane does not exceed $3 a_{0}$. This range is to be compared to $\mathrm{He}-\mathrm{He}$ distances (Fig. 6) ranging from 4 to $15 a_{0}$ and to the $\mathrm{Rb}-\mathrm{He}$ distance (Fig. 5) ranging from 5 to $10 a_{0}$. We found that clusters with small $n$ are quite planar, especially for $n \leq 7$. With increasing cluster size beyond $n$ $=7$, larger out-of-plane motion is observed.

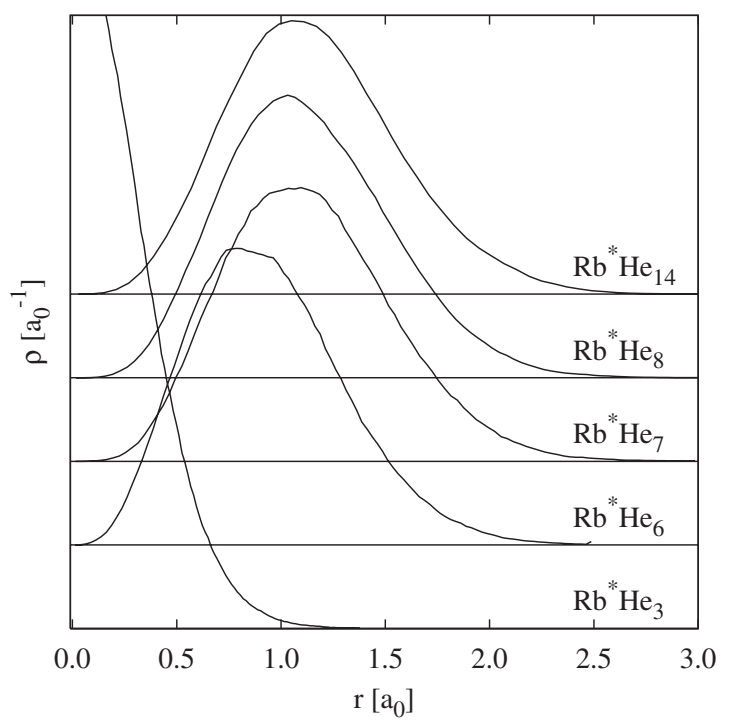

FIG. 9. Distributions of the average distance of He atoms from the mean plane for selected helium clusters vertically shifted for clarity. 


\section{RESULTS FOR Rb ON A ${ }^{4} \mathrm{He}$ SURFACE}

In recent experiments, ${ }^{13} \mathrm{Rb}$ atoms attached to large ${ }^{4} \mathrm{He}$ droplets were excited from the electronic ground state to the $1^{2} \Pi_{1 / 2}$ state. $\mathrm{Rb}$ was found to stay attached to the He surface after relaxing back to the ground state. This implies that $\mathrm{Rb}^{*}$ is also bound to the surface which is in stark contrast to the observation of exciplex formation for excitation to the $1^{2} \Pi_{3 / 2}$ state: in the latter case, the energy released after the formation of $\mathrm{Rb}^{*} \mathrm{He}$ leads to desorption from the droplet. ${ }^{7,9}$ This, in turn, indicates that no exciplex $\mathrm{Rb}^{*} \mathrm{He}_{m}$ is formed upon excitation to the $\Pi_{1 / 2}$ state.

In order to understand these experimental observations, we perform a "vertical Monte Carlo transition," i.e., an abrupt switch of the interaction potential from $\mathrm{Rb}$ to $\mathrm{Rb}^{*}$ in the course of a PIMC simulation. Thus we are able to see how $\mathrm{Rb}^{*} \mathrm{He}_{n}$ equilibrates, starting from $\mathrm{RbHe}_{n}$ configurations. To some extent, this vertical MC transition mirrors the experiment $\mathrm{Rb} \rightarrow \mathrm{Rb}^{*}$, although this PIMC "process" is, of course, not a dynamical simulation of the physical process. PIMC simply relaxes the $\mathrm{Rb}$ adsorption state to the closest (meta-)stable $\mathrm{Rb}^{*}$ adsorption state. Using this approach, we also studied excitation to the higher $\Pi_{3 / 2}$ state of a $\mathrm{Rb}$ atom on the surface of a $100{ }^{4} \mathrm{He}$ atoms cluster. This leads to the formation of a $\mathrm{Rb}^{*} \mathrm{He}$ exciplex ${ }^{55}$ as observed in experiments. $^{7,9}$

A free He surface represents the limit of infinite droplet size. Neither a free He surface nor a very large droplet can be efficiently simulated by QMC. For PIMC simulations, we therefore resort to simulating $\mathrm{Rb}$ on a helium film of finite thickness, adsorbed to layers of "frozen" helium atoms (i.e., $\mathrm{He}$ atoms which are not moved during the simulation). These immobile He atoms are simply providing a substrate potential and must not be confused with real layers of frozen $\mathrm{He}$ which form on sufficiently attractive substrates. For dimples, which are not too deep, a few layers of liquid helium atoms should provide a good approximation to the free surface of an infinite half space of helium or to the surface of a large helium droplet. In the $x$ and $y$ directions parallel to the surface, we apply periodic boundary conditions.

In Fig. 10, we show the profile $\rho_{\mathrm{He}}(z)$ of the film density together with the $\mathrm{Rb}$ and $\mathrm{Rb}^{*}$ density, $\rho_{\mathrm{Rb}}(z)$. The film density (top panel: pure film, middle: $\mathrm{Rb}$, and bottom: $\mathrm{Rb}^{*}$ ) is clearly not affected by the presence of $\mathrm{Rb}$ or $\mathrm{Rb}^{*}$ which means that the surface area is large enough to rule out finite size effects. The probability density for $\mathrm{Rb}$ shown in the middle panel seemingly overlaps with the ${ }^{4} \mathrm{He}$ density because $\mathrm{Rb}$ sits in a deep dimple (see further below). In contrast, $\mathrm{Rb}^{*}$ has relaxed to a metastable weakly bound surface state much farther away from the film (hence sitting in a much shallower dimple, see below). This metastable state is thus very different from the true ground state, which is characterized by a ring-shaped exciplex with seven $\mathrm{He}$ atoms, surrounded by more loosely bound He atoms as studied in Sec. III. In all our PIMC simulations, we converged to a metastable weakly bound state after this vertical MC transition. In particular, we never observed the penetration of $\mathrm{Rb}^{*}$ inside the film or the formation of an exciplex. This means that there is a barrier between this weakly bound state and

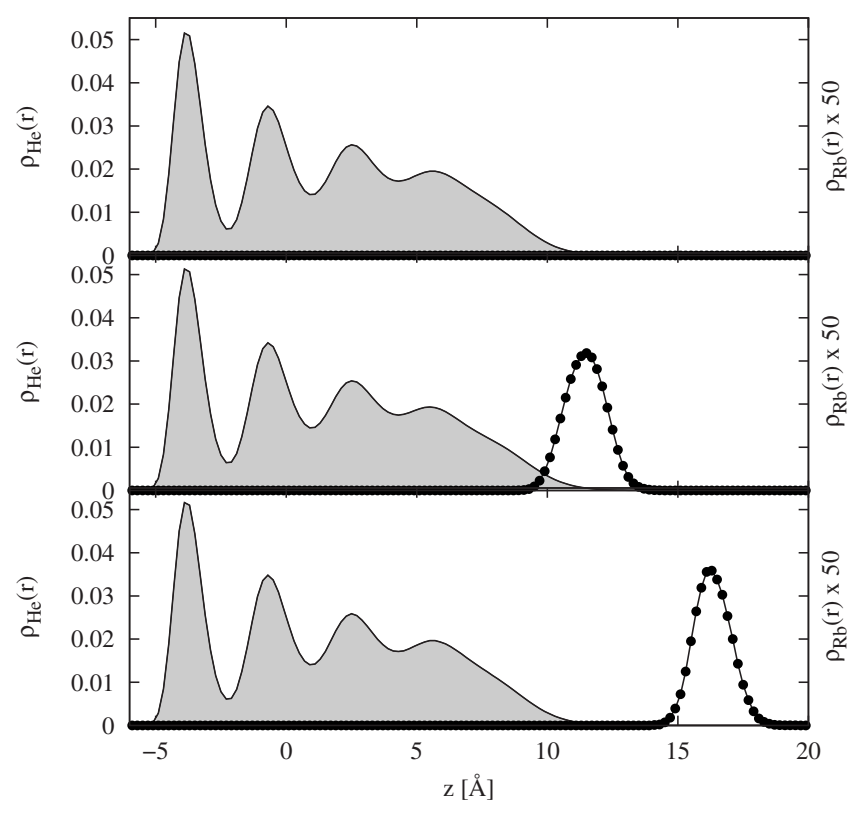

FIG. 10. Density profile (in $\AA^{-2}$ ) of ${ }^{4} \mathrm{He}$ film, pure, and with adsorbed $\mathrm{Rb} / \mathrm{Rb}^{*}$ atom (full line), and density of adsorbed $\mathrm{Rb} / \mathrm{Rb}^{*}$ (line with circles) along $z$. Pure helium case is presented in the top panel, adsorbed $\mathrm{Rb}$ in the middle panel, and adsorbed $\mathrm{Rb}^{*}$ atom in the bottom panel.

the state similar to the ground vibrational state for $\mathrm{Rb}^{*} \mathrm{He}_{n}$ with seven helium atoms closely and strongly bound. This barrier is $32.7 \mathrm{~cm}^{-1}$ in height in the case of $\mathrm{Rb}^{*}-\mathrm{He}$ dimer for which the secondary minimum is only $0.7 \mathrm{~cm}^{-1}$ above the dissociation limit. In the high-dimensional case of $\mathrm{Rb}^{*}$ adsorbed to a He surface, the determination of the barrier height is not easy. A reaction path determination or a transition path sampling could be used to give a classical approximation to the pathway to the formation of the more strongly bound structure but obviously would completely neglect the quantum nature of helium.

In order to address the question of the binding strength of $\mathrm{Rb}^{*}$ to the He film, we have calculated the chemical potential $\mu_{\mathrm{Rb}}=E\left[\mathrm{Rb}^{(*)} \mathrm{He}_{n}\right]-E\left[\mathrm{He}_{n}\right]$ of $\mathrm{Rb}$ and $\mathrm{Rb}^{*}$. The total energy of $\mathrm{He}$ films with and without adsorbed $\mathrm{Rb}^{(*)}$ is tabulated in the first column in Table III; the second column tabulates these energies relative to the pure film, i.e., the chemical potential of $\mathrm{Rb}$ (error bars are about $\pm 1 \mathrm{~K}$ ). $\mathrm{Rb}^{*}$ is bound by only half the energy of Rb. We caution that $\mu_{\mathrm{Rb}}$ is the difference of two large numbers, obtained in two separate simulations; a small inconsistency between them could therefore lead to a large error in the binding energy. Also shown in the table are the average distance $\langle r\rangle$ of $\mathrm{Rb}$ and $\mathrm{Rb}^{*}$ from the

TABLE III. Total energies of pure film and films with adsorbed $\mathrm{Rb}$ and $\mathrm{Rb}^{*}$. The energy difference between the latter and the pure film is the chemical potential $\mu_{\mathrm{Rb}}$. The right column provides the average distance to the surface in $\AA$ (see text for definition).

\begin{tabular}{lccc}
\hline \hline & $\begin{array}{c}\text { Total energy } \\
\left(\mathrm{cm}^{-1}\right)\end{array}$ & $\begin{array}{c}\mu_{\mathrm{Rb}} \\
\left(\mathrm{cm}^{-1}\right)\end{array}$ & $\begin{array}{c}\langle r\rangle \\
(\AA)\end{array}$ \\
\hline Pure film & $-1002.4 \pm 0.3$ & - & - \\
$\mathrm{Rb}$ & $-1013.1 \pm 0.4$ & $-10.8 \pm 0.7$ & 3.4 \\
$\mathrm{Rb}^{*}$ & $-1007.9 \pm 0.3$ & $-5.5 \pm 0.6$ & 8.2 \\
\hline \hline
\end{tabular}




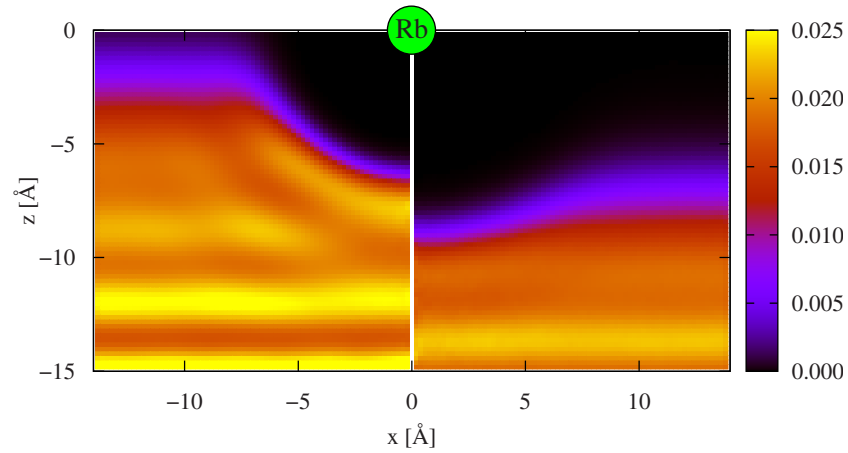

FIG. 11. (Color online) Density distribution of ${ }^{4} \mathrm{He}$ film with respect to adsorbed $\mathrm{Rb}$ (left) and $\mathrm{Rb}^{*}$ (right) atoms, which is located at $(0,0)$.

He surface, which we define as point $z_{0}$, where the density profile has half the bulk equilibrium density, $\rho_{\mathrm{He}}\left(z_{0}\right)$ $=\frac{1}{2} 0.022 \AA^{-2} \cdot \mathrm{Rb}^{*}$ is almost $5 \AA$ farther away from the surface than $\mathrm{Rb}$.

Figure 11 illustrates the dimple caused by the presence of $\mathrm{Rb}$ and $\mathrm{Rb}^{*}$. On the left hand side, the ${ }^{4} \mathrm{He}$ density with respect to the $\mathrm{Rb}$ position is plotted in false color. On the right hand side, the result for $\mathrm{Rb}^{*}$ is shown. The position of $\mathrm{Rb} / \mathrm{Rb}^{*}$ is set to be the origin in the figure, as indicated by the green disk. Apart from confirming that $\mathrm{Rb}^{*}$ indeed sits high above the surface, Fig. 11 illustrates the different dimple shapes that result from such a large $\mathrm{Rb}^{*}$-surface separation: while $\mathrm{Rb}$ generates a very pronounced, deep dimple, the $\mathrm{Rb}^{*}$ dimple is very shallow. In particular, we expect that $\mathrm{Rb}^{*}$ moves more freely parallel to the surface (with an effective mass close to the bare mass) than $\mathrm{Rb}$.

With the above prescription of a vertical MC transition, we cannot make any quantitative statements about the dynamics of the $\mathrm{Rb}$ atom and the $\mathrm{He}$ film after $1^{2} \Sigma_{1 / 2}$ $\rightarrow 1{ }^{2} \Pi_{1 / 2}$ excitation. However we expect that the above arguments for a metastable weakly bound adsorption state holds for the dynamics too. Hence $\mathrm{Rb}^{*}$ is pushed away from the initial dimple formed in the $\Sigma_{1 / 2}$ state. We note, however, that due to the large zero point motion of ${ }^{4} \mathrm{He}$, one should not completely rule out that an exciplex can be formed, albeit with low probability.

Figures 10 and 11 show that the film density has a pronounced layer structure in the vicinity of the adsorbing wall. Since the ground state dimple is quite deep, the layering of the helium film might affect the results. This could be checked by simulations with thicker films, thick ${ }^{4} \mathrm{He}$ slabs or very large ${ }^{4} \mathrm{He}$ droplets all of which are computationally too demanding. Alternatively, we are considering hypernettedchain/Euler-Lagrange calculations similar to the ones in Ref. 56.

Why not perform MC simulations of the full dynamic process? MC methods can only sample equilibrium or metastable configurations; thus MC can describe dynamic processes only in the linear response regime because linear response theory is formulated in terms of time-dependent correlation functions in equilibrium. However, for retrieving real-time correlation functions, an inverse Laplace transformation has to be performed. ${ }^{57}$ This approach was successfully applied, for example, to rotational dynamics of mol- ecules in ${ }^{4} \mathrm{He}$ clusters. ${ }^{53}$ However, the inverse Laplace transformation is an ill-conditioned problem, which requires performing extremely time consuming MC simulations which in our case is exacerbated by the slower sampling of the heavy $\mathrm{Rb}$ motion and the large number of $\mathrm{He}$ atoms that are needed to form a well-defined surface. Even if computational cost would not be a concern, full reconstruction of the real-time correlations would not be possible. Finally, it is questionable if linear response theory is applicable to the process of electronic excitation. For all these reasons, in this work we refrained from this approach.

\section{CONCLUSIONS}

Helium-4 droplets have become an important tool of matrix spectroscopy. Experimental studies of alkali atoms in solid ${ }^{4} \mathrm{He}$ and on the surface of helium clusters have lead to interesting insights and have posed new questions regarding exciplex formation (as mentioned in Sec. I), formation of small alkali clusters such as trimers,${ }^{58}$ and even the possibility of an unusual solid state of ${ }^{4} \mathrm{He}$ doped with alkali atoms. ${ }^{59}$ In this work we have focused on the electronic excitation of a single $\mathrm{Rb}$ atom in ${ }^{4} \mathrm{He}$ environment using diffusion and PIMC methods.

Using a DIM approach to model the full interaction potential, we have first built up small $\mathrm{Rb}^{*} \mathrm{He}_{n}$ clusters by successively adding $\mathrm{He}$ atoms to an $\mathrm{Rb}^{*}$ atom in the excited state $1{ }^{2} \Pi_{1 / 2}$. Up to $n=7 \mathrm{He}$ atoms, we obtained strongly bound, exciplexlike clusters where the He atoms form a ring around $\mathrm{Rb}^{*}$. In the $n=7$ cluster, He forms a particularly rigid ring, as seen in the angular $\mathrm{He}-\mathrm{He}$ distribution function. Additional ${ }^{4} \mathrm{He}$ atoms cannot be accommodated in the ring close to $\mathrm{Rb}^{*}$, and a much more diffuse torus is formed around the rigid seven-membered ring, i.e., the growth of the cluster beyond the core ring is still in a plane. The formation of such anisotropic clusters highlights the role of the nonpairwise additive $\mathrm{Rb}^{*}-\mathrm{He}_{n}$ interaction-a pairwise additive potential could only lead to isotropic clusters.

Our second line of investigation concerns the excitation of $\mathrm{Rb}$ sitting on the surface of liquid ${ }^{4} \mathrm{He}$, modeled by a film, from the electronic ground state to the ${ }^{2} \Pi_{1 / 2}$ state. We performed several "vertical MC transitions" where, starting from the equilibrated configuration of $\mathrm{Rb}$ in a surface dimple, we switch to the $\mathrm{Rb}^{*}-\mathrm{He}_{n}$ potential. We found that $\mathrm{Rb}^{*}$ equilibrates to a metastable weakly bound state in a very shallow dimple and did not find evidence for the formation of an exciplex with one or more ${ }^{4} \mathrm{He}$ atoms. This finding is consistent with the recent experimental results ${ }^{13}$ and very different to the exciplex formation observed for $\mathrm{Rb}$ embedded in solid helium-4. ${ }^{10,23}$

With the QMC methods used in this work, further related problems can be studied. We are investigating $\mathrm{Rb}^{*} \mathrm{He}_{n}$ in the $1^{2} \Pi_{3 / 2}$ state, where the formation of $\mathrm{Rb}^{*} \mathrm{He}$ exciplexes has been observed, ${ }^{7}$ as well as $\mathrm{Rb}_{2}$ dimers in the triplet state, as studied experimentally in Ref. 60. We note that QMC is an equilibrium method, and the study of the dynamics of excitations of atom in a superfluid or solid ${ }^{4} \mathrm{He}$ matrix is a great theoretical challenge. Some progress has been made by applying a phenomenological approach using density func- 
tional theory, ${ }^{20}$ which reduces the many-body problem of the ${ }^{4} \mathrm{He}$ environment to an effective one-body problem that allows calculation of dynamical properties. We are planning to study the coupled dynamics of $\mathrm{Rb}$ and the ${ }^{4} \mathrm{He}$ surface upon excitation to $1^{2} \Pi_{1 / 2}$ using correlated basis function theory, ${ }^{61}$ taking into account also energy dissipation via collective excitations of helium.

\section{ACKNOWLEDGMENTS}

We thank Professor J. Pascale for making the alkalihelium interaction potentials available to us and Professor W. Ernst and Professor F. Stienkemeier for valuable discussions. This work is supported by Egide via the Amadeus/Amadée PHC program No. 17283PE/FR142008. M.L. acknowledges grants from CNRS and from the Academy of Finland.

${ }^{1}$ Helium Nanodroplets: A Novel Medium for Chemistry and Physics, special issue of J. Chem. Phys. 115(22) (2001)

${ }^{2}$ J. P. Toennies and A. F. Vilesov, Angew. Chem., Int. Ed. 43, 2622 (2004)

${ }^{3}$ M. Barranco, R. Guardiola, S. Hernandez, R. Mayol, J. Navarro, and M. Pi, J. Low Temp. Phys. 142, 1 (2006).

${ }^{4}$ F. Stienkemeier and K. K. Lehmann, J. Phys. B 39, R127 (2006).

${ }^{5}$ M. Hartmann, A. Lindinger, J. P. Toennies, and A. F. Vilesov, Phys. Chem. Chem. Phys. 4, 4839 (2002).

${ }^{6}$ H. Whitley, P. Huang, Y. Kwon, and K. B. Whaley, J. Chem. Phys. 123, 054307 (2005).

${ }^{7}$ F. R. Brühl, R. A. Trasca, and W. E. Ernst, J. Chem. Phys. 115, 10220 (2001).

${ }^{8}$ C. P. Schulz, P. Claas, and F. Stienkemeier, Phys. Rev. Lett. 87, 153401 (2001).

${ }^{9}$ G. Droppelmann, O. Büermann, C. P. Schulz, and F. Stienkemeier, Phys. Rev. Lett. 93, 023402 (2004)

${ }^{10}$ A. Hofer, P. Moroshkin, D. Nettels, S. Ulzega, and A. Weis, Phys. Rev. A 74, 032509 (2006).

${ }^{11}$ J. Reho, J. Higgins, K. K. Lehmann, and G. Scoles, J. Chem. Phys. 113, 9694 (2000).

${ }^{12}$ J. Reho, J. Higgins, C. Callegari, K. K. Lehmann, and G. Scoles, J. Chem. Phys. 113, 9686 (2000)

${ }^{13}$ G. Auböck, J. Nagl, C. Callegari, and W. E. Ernst, Phys. Rev. Lett. 101, 035301 (2008)

${ }^{14}$ O. Bünermann, M. Mudrich, M. Weidemüller, and F. Stinkemeier, J. Chem. Phys. 121, 8880 (2004).

${ }^{15}$ F. Stienkemeier, J. Higgins, C. Callegari, S. I. Kanorsky, W. E. Ernst, and G. Scoles, Z. Phys. D: At., Mol. Clusters 38, 253 (1996).

${ }^{16}$ C. Callegari, J. Higgins, F. Stienkemeier, and G. Scoles, J. Phys. Chem. A 102, 95 (1998).

${ }^{17}$ F. Ancilotto, G. DeToffol, and F. Toigo, Phys. Rev. B 52, 16125 (1995).

${ }^{18}$ A. Nakayama and K. Yamashita, J. Chem. Phys. 114, 780 (2001).

${ }^{19}$ J. Dupont-Roc, Z. Phys. B: Condens. Matter 98, 383 (1995).

${ }^{20}$ G. DeToffol, F. Ancilotto, and F. Toigo, J. Low Temp. Phys. 102, 381 (1996).

${ }^{21}$ D. Nettels, A. Hofer, P. Moroshikin, R. Müller-Siebert, S. Ulzega, and A. Weis, Phys. Rev. Lett. 94, 063001 (2005).

${ }^{22}$ P. Moroshkin, A. Hofer, D. Nettels, S. Ulzega, and A. Weis, J. Chem. Phys. 124, 024511 (2006).

${ }^{23}$ P. Moroshkin, A. Hofer, S. Ulzega, and A. Weis, Low Temp. Phys. 32
981 (2006).

${ }^{24}$ K. Enomoto, K. Hirano, M. Kumakura, Y. Takahashi, and T. Yabuzaki, Phys. Rev. A 69, 012501 (2004).

${ }^{25}$ K. Enomoto, M. Kumakura, Y. Takahashi, and T. Yabuzaki, Phys. Rev. A 66, 042505 (2002)

${ }^{26}$ T. Takayanagi and M. Shiga, Phys. Chem. Chem. Phys. 6, 3241 (2004).

${ }^{27}$ R. A. Aziz, F. R. W. McCourt, and C. C. K. Wong, Mol. Phys. 61, 1487 (1987).

${ }^{28}$ J. Pascale, Service de Physique des Atomes et des Surfaces (C.E.N. Saclay), Gif sur Yvette, France, 1983.

${ }^{29}$ F. O. Ellison, J. Am. Chem. Soc. 85, 3540 (1963).

${ }^{30}$ J. Pascale, Phys. Rev. A 28, 632 (1983).

${ }^{31}$ K. Hirano, K. Enomoto, M. Kumakura, Y. Takahashi, and T. Yabuzaki, Phys. Rev. A 68, 012722 (2003).

${ }^{32}$ M. Mudrich, F. Stienkemeier, G. Droppelmann, P. Claas, and C. P. Schulz, Phys. Rev. Lett. 100, 023401 (2008).

${ }^{33}$ H. A. Kramers, Proc. R. Acad. Sci. Amsterdam 33, 959 (1930).

${ }^{34}$ P. J. Reynolds, D. M. Ceperley, B. J. Alder, and W. A. Lester, J. Chem. Phys. 77, 5593 (1982)

${ }^{35}$ M. A. Suhm and R. O. Watts, Phys. Rep. 204, 293 (1991).

${ }^{36}$ V. Buch, J. Chem. Phys. 97, 726 (1992).

${ }^{37}$ C. J. Umrigar, M. P. Nightingale, and K. J. Runge, J. Chem. Phys. 99, 2865 (1993).

${ }^{38}$ B. L. Hammond, W. A. Lester, and P. J. Reynolds, Monte Carlo Methods in Ab Initio Quantum Chemistry (World Scientific, Singapore, 1994).

${ }^{39}$ A. Viel, M. V. Patel, P. Niyaz, and K. B. Whaley, Comput. Phys. Commun. 145, 24 (2002).

${ }^{40} \mathrm{M}$. Lewerenz, personal communication (2006).

${ }^{41}$ D. Blume, M. Lewerenz, F. Huisken, and M. Kaloudis, J. Chem. Phys. 105, 8666 (1996).

${ }^{42}$ A. Viel, M. D. Coutinho-Neto, and U. Manthe, J. Chem. Phys. 126, 024308 (2007).

${ }^{43}$ J. Casulleras and J. Boronat, Phys. Rev. B 52, 3654 (1995).

${ }^{44}$ G. L. Warren and R. J. Hinde, Phys. Rev. E 73, 056706 (2006).

${ }^{45}$ C. A. Brindle, M. R. Prado, K. C. Janda, N. Halberstadt, and M. Lewerenz, J. Chem. Phys. 123, 064312 (2005)

${ }^{46}$ R. P. Feynman and A. R. Hibbs, Quantum Mechanics and Path Integrals, International Series in Pure and Applied Physics (McGraw-Hill, New York, 1965).

${ }^{47}$ D. Chandler and P. G. Wolynes, J. Chem. Phys. 74, 4078 (1981).

${ }^{48}$ N. Metropolis, A. W. Rosenbluth, M. N. Rosenbluth, A. H. Teller, and E. Teller, J. Chem. Phys. 21, 1087 (1953).

${ }^{49}$ S. A. Chin, Phys. Lett. A 226, 344 (1997).

${ }^{50}$ S. A. Chin, Phys. Rev. E 73, 026705 (2006).

${ }^{51}$ D. M. Ceperley, Rev. Mod. Phys. 67, 279 (1995).

${ }^{52}$ N. Blinov, X. Song, and P. N. Roy, J. Chem. Phys. 120, 5916 (2004).

${ }^{53}$ R. E. Zillich, F. Paesani, Y. Kwon, and K. B. Whaley, J. Chem. Phys. 123, 114301 (2005).

${ }^{54}$ S. Baroni and S. Moroni, Comput. Phys. Commun. 6, 1884 (2005).

${ }^{55}$ R. E. Zillich, M. Leino, and A. Viel (unpublished).

${ }^{56}$ E. Krotscheck and R. Zillich, Phys. Rev. B 77, 094507 (2008).

${ }^{57}$ M. Boninsegni and D. M. Ceperley, J. Low Temp. Phys. 104, 339 (1996)

${ }^{58}$ J. Nagl, G. Auböck, A. W. Hauser, O. Allard, C. Callegari, and W. E. Ernst, Phys. Rev. Lett. 100, 063001 (2008).

${ }^{59}$ P. Moroshkin, A. Hofer, S. Ulzega, and A. Weis, Nat. Phys. 3, 786 (2007).

${ }^{60}$ G. Auböck, J. Nagl, C. Callegari, and W. E. Ernst, J. Phys. Chem. A 111, 7404 (2007)

${ }^{61}$ R. E. Zillich and K. B. Whaley, Phys. Rev. B 69, 104517 (2004). 